\title{
Genetic linkage analysis of pulmonary fibrotic response to silica in mice
}

\author{
Y. Ohtsuka, X-T. Wang, J. Saito, T. Ishida and M. Munakata
}

ABSTRACT: Inter-individual variations in the development of silicosis, even within the same environments, have been reported, which suggest the contribution of genetic factors in silicosis aetiology. The aim of the present study was to determine whether there is any significant genetic influence on the development of silicosis. Furthermore, which genetic loci are responsible for the pulmonary response to silica exposure?

Eight strains of inbred mice were used to examine the genetic influence on the lung fibrotic response to silica exposure. After intercross-breeding between the most susceptible and most resistant strains, a genome-wide linkage analysis of quantitative trait loci (QTL) was performed. Hydroxyproline was applied as an index, and genotypes of 167 marker genes were analysed by fragment analysis using a capillary-type sequencer.

There was significant inter-strain difference in the mean concentration of hydroxyproline contents among the eight strains of mice. Breeding studies were conducted between the most susceptible, C57BL/6J, and the most resistant strain, CBA/J. A genome-wide linkage analysis of silica-exposed intercrossed cohorts identified significant QTL on chromosome 4 and suggestive QTL on chromosomes 3 and 18.

The present study demonstrates that genetic factors may play a significant role in fibrotic-lung responses to silica; one significant and two suggestive quantitative trait loci were identified.

KEYWORDS: C57BL/6J, CBA/J, hydroxyproline, intercross, quantitative trait locus

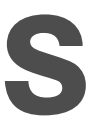
ilicosis is an occupational lung disease caused by inhalation of dust containing free crystalline silica. Free-silica is still an occupational fibrogenic hazard despite the technical adequacy of current protective equipment. In the USA, it was estimated that 200,000 miners and 1.7 million nonmining workers had occupational exposures to inhaled silica [1]. In the advanced stage, silicosis complicates tuberculosis, respiratory failure due to progressive massive fibrosis, emphysema, and lung cancer. Considerable attention has been paid to these adverse effects of silica dusts.

Concerning the development of silicosis, extrinsic factors such as duration, amount of exposure, and content of free crystalline silica have been known as critical determinants of the progression of silicosis [2]. However, some of the intrinsic (genetic) factors that may influence susceptibility to silicosis have been reported. Associations of specific human leukocyte antigen (HLA) haplotype with silicosis have been reported in a Japanese population [3]. In a German population, HLA haplotype association with coal worker's pneumoconiosis has also been reported [4]. Associations of gene polymorphisms of pro-inflammatory cytokines (e.g. tumour necrosis factor- $\alpha$, interleukin-1 receptor) have been reported [5-7]. In addition to these epidemiological studies, the present authors clarified the importance of genetic background on the variation in inflammation and fibrotic response in a murine silicosis model $[8,9]$.

The objective of the current study was to identify, using inbred mice, the genetic basis for susceptibility to silica-induced pulmonary fibrosis. Inbred mice provide a well-characterised animal model for understanding genetic and environmental factors that determine susceptibility to complex disease processes. Furthermore, the mouse is accepted as an excellent model of human disease processes because there is extensive linkage homology between the mouse and human genomes.

Any genetic influence on the inflammatory and fibrotic response to silica was examined in eight strains of inbred mice. A genome-wide search for genes responsible for silicosis was also conducted in the present model. The current study reports significant genetic influence on the fibrotic response among eight strains of mice compared

\section{AFFILIATIONS}

Dept of Pulmonary Medicine, School of Medicine, Fukushima Medical University, Fukushima, Japan.

CORRESPONDENCE

Y. Ohtsuka

Dept of Internal Medicine Iwamizawa Rosai Hospital 4-jyo

Higashi 16-chome 5

Iwamizawa

Hokkaido 068-0004

Japan

Fax: 81126221304

E-mail: yohtsuka@kvf.biglobe.ne.jp

Received:

November 112005

Accepted after revision:

June 292006

SUPPORT STATEMENT

The present study was supported by research grants from the Ministry of Education, Science and Culture, Japan (№. 13470129). 
with titanium dioxide $\left(\mathrm{TiO}_{2}\right)$-instilled control, and the linkage analysis of intercrossed (F2) mice identified significant quantitative trait loci (QTL) on chromosome 4 and suggestive QTL on chromosomes 3 and 18. Some of the results of the current study have been previously reported [9].

\section{MATERIALS AND METHODS Animals}

Male (6-8 week old) mice of the following inbred strains were purchased from Sankyo Labo Service (Tokyo, Japan) and Clea Japan Inc. (Tokyo, Japan): A/J, AKR/J, Balb/cJ, C3H/HeJ, C57BL/6J, C57BL/10J, CBA/J, and DBA/2J. Breeding studies between the most susceptible and most resistant strains of mice were conducted. The following crosses were generated: male F1 were bred with females of the most susceptible strain to produce backcross progeny; F1 were intercrossed to produce F2 progeny. Progeny were weaned at 4 weeks of age, separated according to sex and housed in micro-isolation cages until they reached the appropriate age for experimentation (6-8 weeks). The mice were handled in accordance with the Helsinki convention and the standards established by the Animal Welfare Acts set forth by Fukushima Medical University guidelines.

\section{Study design}

Inter-strain comparison

Mice of all eight strains aged 6-8 weeks were anesthetised with i.p. pentobarbital and their trachea exposed surgically. Prepared silica $\left(0.2 \mathrm{~g} \cdot \mathrm{kg}^{-1}\right.$ in $50 \mathrm{mg} \cdot \mathrm{mL}^{-1}$ saline $)$ was instilled intratracheally, as described in previously [8]. The control animals in each strain were given $0.2 \mathrm{~g} \cdot \mathrm{kg}^{-1}$ of inert $\mathrm{TiO}_{2}$ $\left(50 \mathrm{mg} \cdot \mathrm{mL}^{-1}\right.$ saline) intratracheally. Twenty-eight days after intratracheal silica instillation, pathological fibrosis indices were evaluated using estimates by AsHCROFT et al. [10], and hydroxyproline content per lung was also assessed.

\section{Linkage analysis}

Intercross (F2) mice (6-8 weeks old) were anesthetised and exposed to silica as described above. As a control, three mice of the most susceptible strain were given $0.02 \mathrm{~mL}$ of intratracheal saline in each experiment. Twenty-eight days after silica instillation, collagen deposition of the right lung in each mouse was assayed and the kidneys taken for DNA extraction.

\section{Collagen assay}

Collagen deposition was estimated by determining the total hydroxyproline content of the left lung. Hydroxyproline was measured according to a method by WOESSNER [11] as reported previously [8]. Results were calculated as micrograms hydroxyproline per lung $\left(\mu \mathrm{g} \cdot \mathrm{lung}^{-1}\right)$, and expressed as the ratio of the mean value of control mice in each experiment.

\section{Morphological evaluation of lung sections}

The right lungs were fixed, embedded in paraffin, sectioned horizontally to include most of the parenchyma at $4 \mu \mathrm{m}$, and stained with haematoxylin-eosin. The sections were scanned, by an observer blind to treatment, to determine the degree of fibrosis in 30 fields per sample at $100 \times$ magnification and analysed semi-quantitatively according to the method by AsHCROFT et al. [10]. Values of the samples were expressed as a fibrosis index, the mean grade of fibrosis per field.

\section{DNA extraction and genotyping}

DNA was extracted from a kidney of each phenotyped animal and prepared for PCR. PCR reactions were run in 96-well plates with $15.0 \mu \mathrm{L}$ total volume consisting of: $1 \mu \mathrm{L}$ DNA (4 ng); $1.5 \mu \mathrm{L} 10 \times$ reaction buffer; $1.5 \mu \mathrm{L} 25 \mathrm{mM} \mathrm{MgCl}_{2} ; 1.5 \mu \mathrm{L}$ $2.5 \mathrm{mM}$ deoxynucleotide triphosphates (equal mixture of deoxy-adenosine, -cytidine, -guanosine and -thymidine triphosphates); $0.3 \mu \mathrm{L} 10 \mathrm{mM}$ primers (Applied Biosystems, Foster city, CA, USA); 0.6 U Taq polymerase; and brought to volume with distilled water. Primers for simple sequence-length polymorphisms (SSLPs) that differed between C57BL/6J and CBA/J progenitors were chosen from a website [12].

PCR amplification consisted of 10 cycles $\left(94^{\circ} \mathrm{C}\right.$ for $15 \mathrm{~s}, 55^{\circ} \mathrm{C}$ for $15 \mathrm{~s}$ and $72^{\circ} \mathrm{C}$ for $30 \mathrm{~s}$ ) preceded by a denaturation step of $94^{\circ} \mathrm{C}$ for $12 \mathrm{~min}$, and followed by 20 cycles $\left(89^{\circ} \mathrm{C}\right.$ for $15 \mathrm{~s}, 55^{\circ} \mathrm{C}$ for $15 \mathrm{~s}$, and $72^{\circ} \mathrm{C}$ for $30 \mathrm{~s}$ ) and a final elongation step for $10 \mathrm{~min}$ at $72^{\circ} \mathrm{C}$. Pooled PCR product was mixed with 310 Genetic analyser standard solution (Gene Scan 400HD ROX $0.5 \mu \mathrm{L}$, deionised formamide $12 \mu \mathrm{L}$ ), and denatured at $95^{\circ} \mathrm{C}$ for $2 \mathrm{~min}$. This sample was quickly chilled on ice until loading on the machine. The treated PCR products were analysed by ABI PRISM 310 Genetic Analyser with the Gene Scan program (Applied Biosystems).

\section{Statistical analysis of inter-strain comparison}

The effects of exposure (silica versus $\mathrm{TiO}_{2}$ ) and strain on pulmonary responses were assessed by two-way ANOVA [13]. Tukey's test was used for a posteriori comparisons of means. In a posteriori comparison of hydroxyproline content among silicaexposed C57BL/6J, CBA/J, and B6CBAF1/J mice, Dunnett's T3-test was used because of the different number of mice in each group [13]. The strain distribution patterns (SDPs) for lung responses to silica were compared with the SDPs for silica by nonparametric Spearman rank-correlation. Significance was accepted at $\mathrm{p}<0.05$.

\section{Linkage analysis}

A scan of the entire genome was completed by genotyping 25 phenotypically high-responder $(n=13)$ and nonresponder $(n=12)$ mice from a total population of 117 F2 mice [13]. Interval analyses were performed for each SSLP marker and at 10-centimorgan intervals between SSLPs. The dominance properties of each putative QTL were evaluated by performing interval analyses using free, additive, recessive, and dominant regression models. The regressions and significance of each association, likelihood ratio Chi-squared statistic, were calculated. Putative QTLs were further analysed by including the entire F2 cohort within the chromosomes identified by selective genotyping. Permutation tests were performed on the phenotype and the genotype data to establish empirically the significance thresholds of all QTL mapping results, using Map Manager QTX [14] and following the methods of Churchill and Doerge [15]. For the genome scan, 10,000 permutations were performed to establish significant and suggestive linkage threshold values. These values correspond to the genome-wide probabilities proposed by LANDER and KRUGLYAK [16].

\section{RESULTS}

Hydroxyproline content per lung and pathological fibrosis indices were used as indicators of lung fibrosis. ANOVA of 


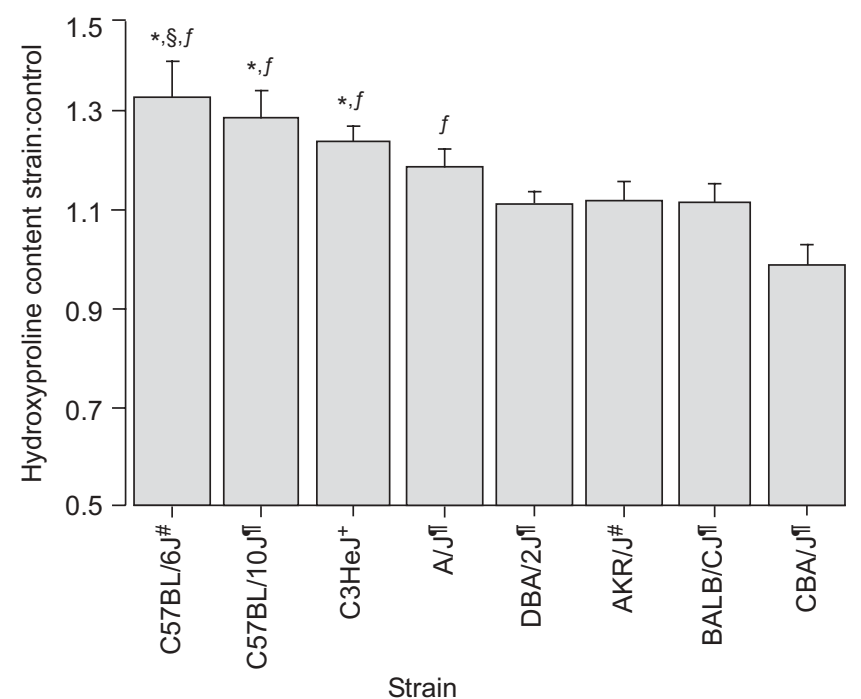

FIGURE 1. The ratio of lung hydroxyproline content in eight mice strains 28 days after intratracheal silica instillation. Data are presented as mean+SE. ${ }^{\#}: n=8 ;$; $\mathrm{n}=12 ;{ }^{+}: \mathrm{n}=16 ;^{\text {s. }}$ : significantly different to Balb/cJ; ${ }^{\text {: }}$ significantly different to CBA; *: $p<0.05$ versus control.

both indicators indicated significant effects of strain $(\mathrm{p}<0.001$ and $\mathrm{p}<0.0025$, respectively) and exposure $(\mathrm{p}<0.0005$, $p<0.0005$ ), and interaction of strain and exposure effects on the mean ratio of hydroxyproline in silica-exposed groups $(\mathrm{p}<0.0005)$ and pathological fibrosis indices $(\mathrm{p}<0.0005)$ compared with $\mathrm{TiO}_{2}$ exposed mice.

The strain distribution pattern in hydroxyproline contents among the eight strains of silica-exposed mice are shown in figure 1 . The hydroxyproline content in C57BL/6J was the highest and in CBA/J was the lowest among eight strains of mice. Multiple comparisons by Tukey's test revealed significant inter-strain differences in the fibrotic response. Significant inter-strain differences were detected between C57BL/6J and $\mathrm{Balb} / \mathrm{cJ}(\mathrm{p}<0.05)$, and between C57BL/6J and CBA/J $(\mathrm{p}<0.05)$. The hydroxyproline content of all other silica-exposed strains, except CBA/J mice, were significantly higher than that of silica-exposed CBA/J $(\mathrm{p}<0.05)$.

The strain distribution pattern in pathological fibrosis indices among the eight strains of silica-exposed mice is shown in figure 2. The pathological fibrosis index in $\mathrm{A} / \mathrm{J}$ was the highest, and that in CBA/J was the lowest among eight strains of mice. Multiple comparisons by Tukey's test revealed significant inter-strain differences in the fibrotic response. Significant inter-strain differences were detected among $\mathrm{A} / \mathrm{J}$, $\mathrm{AKR} / \mathrm{J}$, and CBA $/ \mathrm{J}(\mathrm{p}<0.05)$. The pathological fibrosis indices of all other silica-exposed strains except $\mathrm{CBA} / \mathrm{J}$ mice were significantly higher than that of silica-exposed CBA/J $(\mathrm{p}<0.05)$.

As any phenotype chosen for linkage analysis investigation must be highly reproducible and quantitative [17], hydroxyproline was chosen as a marker of pulmonary fibrosis. From the study results of hydroxyproline, C57BL/6 $\mathrm{J}$ was named as a susceptible strain and $\mathrm{CBA} / \mathrm{J}$ as a resistant strain. To

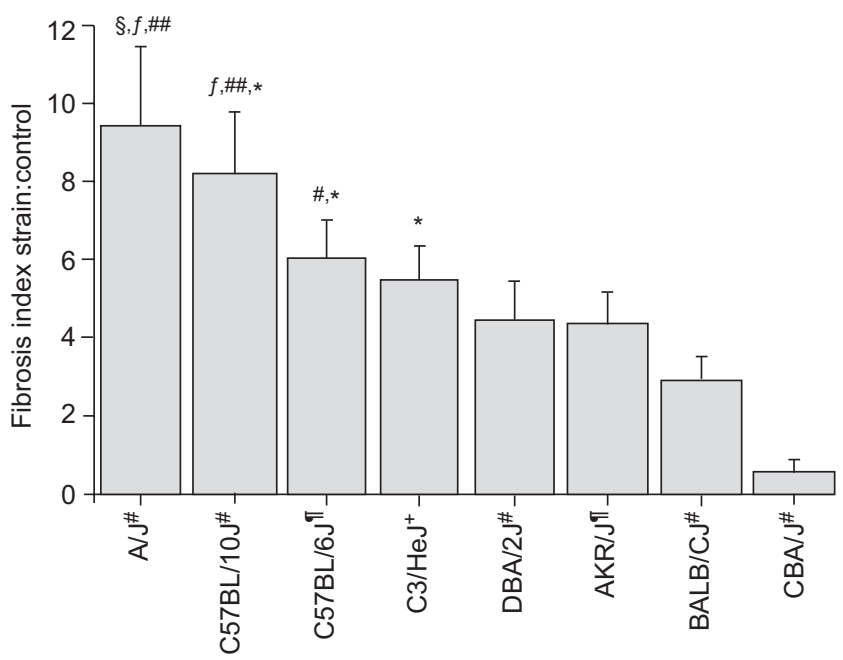

Strain

FIGURE 2. Fibrosis indices in eight strains of mice 28 days after intratracheal silica injection. Values are mean+SE. ${ }^{\#}: n=12 ;: n=8 ;{ }^{+}: n=16 ;{ }^{\S}$ : significantly different to AKR; ${ }^{f}$ : significantly different to Balb/cJ; \#\#: significantly different to CBA. *: significant increase in fibrosis compared to own control $p<0.05$.

obtain insight about the mode of inheritance of susceptibility to silica-exposure, F1 mice derived from a cross between susceptible C57BL/6J and resistant CBA/J mice were phenotyped for their response to silica exposure. Compared with $\mathrm{TiO}_{2}$-exposed control animals, the range of responses to silica exposure by $\mathrm{F} 1$ mice was between that of C57BL/6J and CBA/J progenitor mice (fig. 3). The mean response of $\mathrm{F} 1$ mice was significantly different from those of C57BL/6J and CBA/J mice $(\mathrm{p}<0.05)$.

A genome-wide linkage analysis was initiated to identify the chromosomal location of QTL, which control susceptibility to intratracheal silica exposure. In order to minimise experimental error [18], saline was used as a control for silica-instillation in the linkage analysis study. The first step in the analysis was to select 25 phenotypically extreme F2 responders (50 meioses), genotyped at 167 SSLP markers spaced to provide complete coverage of the mouse genome with $95 \%$ confidence $[12,14]$. Interval analyses with free regression model revealed eight suggestive QTLs on chromosomes 3 (D3mit57.1), 4 (D4Mit9.1) 5 (D5Mit309.1, D5Mit277.1), 6 (D6Mit274.1), 10 (D10Mit230.1), 11 (D11Mit289.1), 14 (D14Mit98.1), and 18 (D18Mit177.1; fig. 4).

Each putative QTL was analysed further by including the F2 cohort ( $\mathrm{n}=117$ animals, 234 meioses). Only the chromosome 4 QTL revealed statistically significant linkage as determined empirically by permutation test (fig. 5). The amount of total trait variance explained by this QTL (at D4Mit9.1) was $\sim 9 \%$. The QTL on chromosomes 3 and 18 exceeded the threshold for suggestive linkage after the remaining F2 animals were considered in the analysis. The amount of total trait variance explained by these QTL, at D3Mit319 and D18Mit177.1, was $\sim 7$ and $6 \%$, respectively. Further interval analyses were not performed, due to the unavailability of commercial SSLPs between C57BL/6J and CBA/J. 

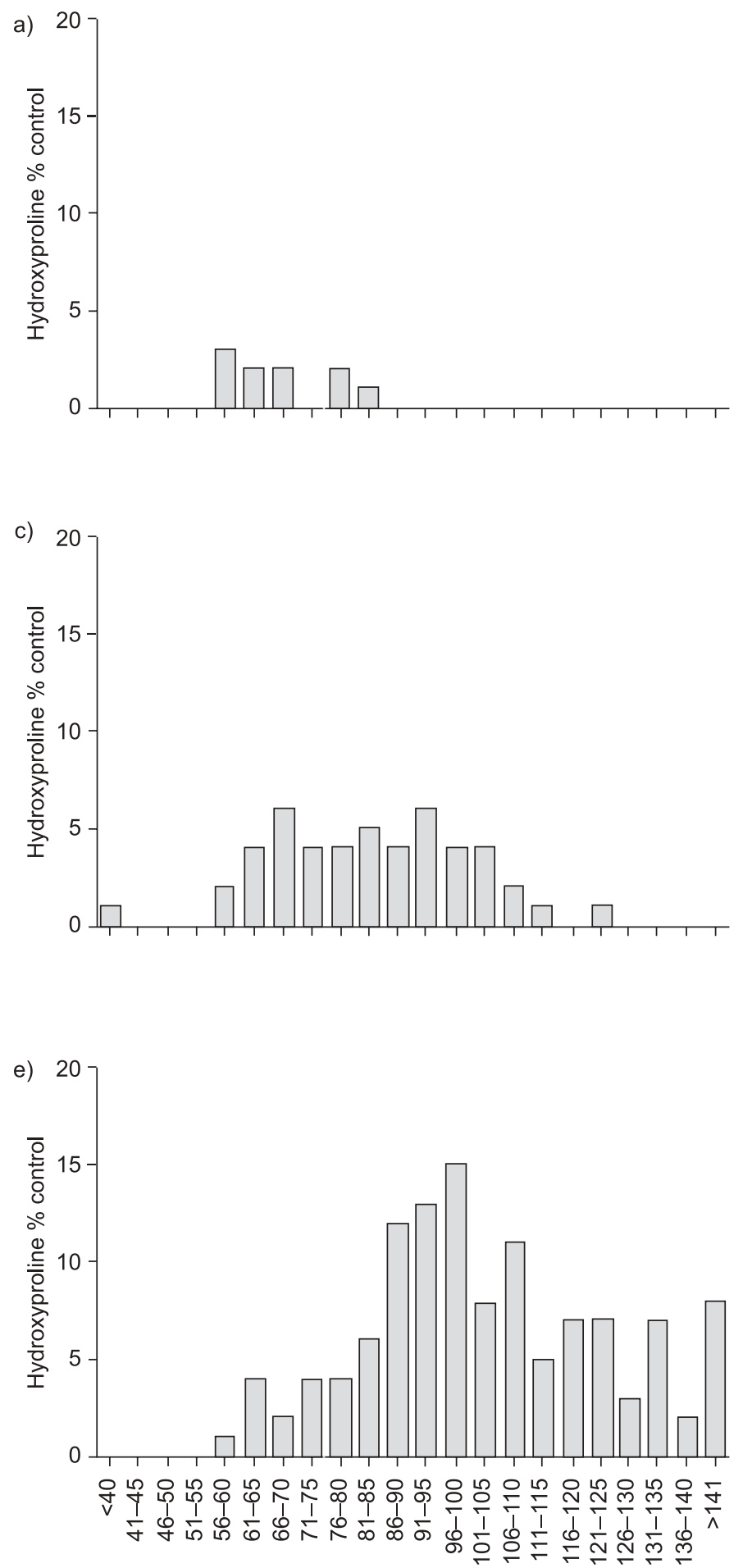

Frequency

\section{DISCUSSION}

A clear pathological association between exposure to silica particles and silicosis has been clarified since antiquity. Epidemiological studies have also shown that duration and amount of exposure as well as content of free crystalline silica are most critical determinants of the progression of silicosis [2]. However, inter-individual variations in the development of b)

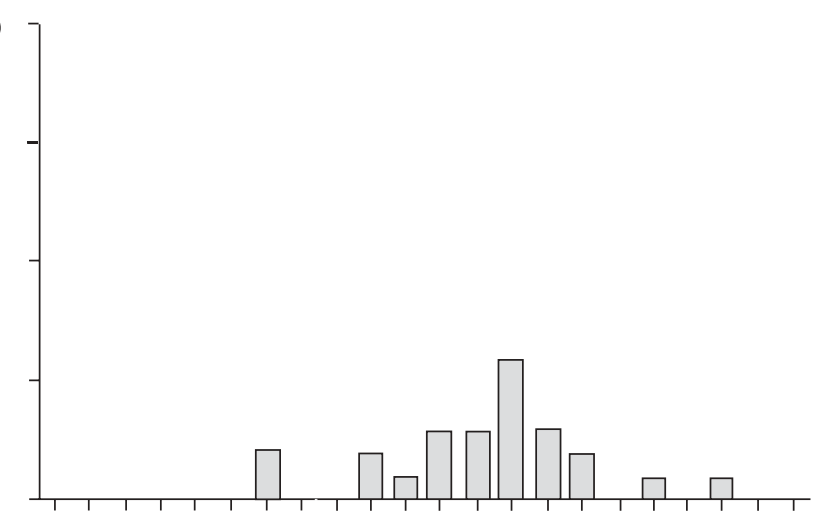

d)

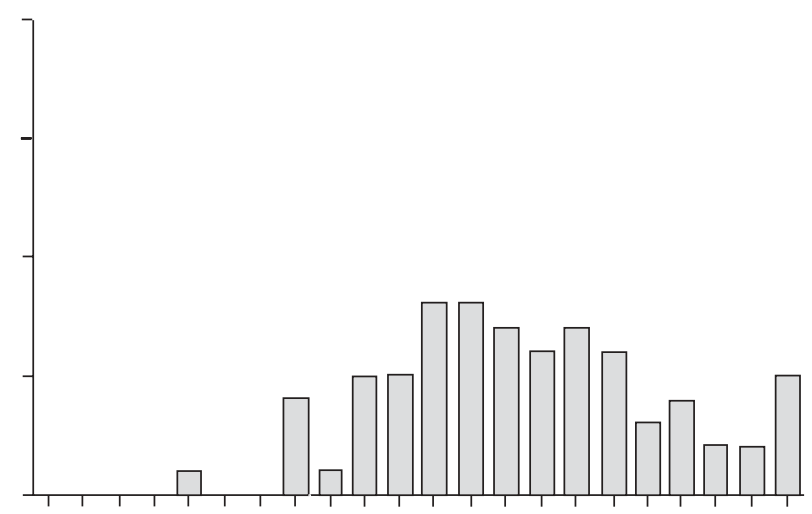

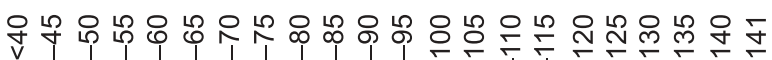

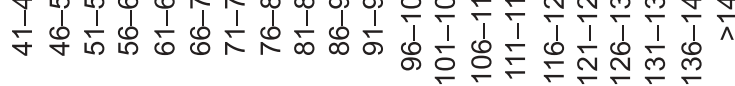

Frequency
FIGURE 3. Frequency distribution of the hydroxyproline content per lung (\% of control) from a) CBA/J mice $(n=10)$ and b) C57BL/6J mice $(n=23)$ and their progeny. Male CBA/J mice were bred with female C57BL/6 to produce $\mathrm{c}$ ) B6CBAF1 hybrids ( $n=48$ ). Male B6CBAF1 mice were bred with C57BL/6J females to produce d) B6BX mice $(n=74)$. B6BX mice were then intercrossed to produce e) B6CBAF2 $(n=119)$. 

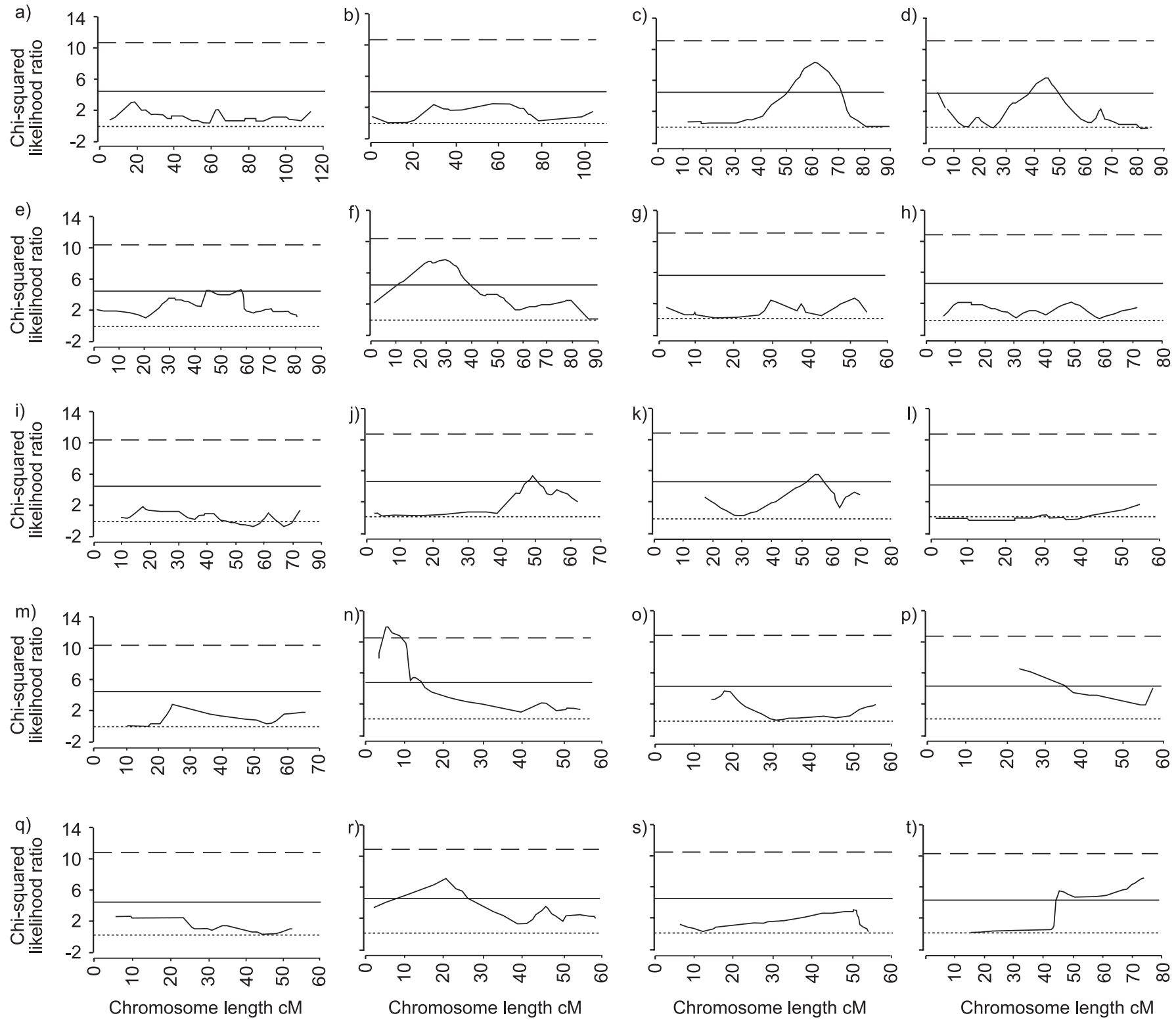

FIGURE 4. A genome-wide search for quantitative trait loci (QTL) by selective genotyping of the $25 \mathrm{~F} 2$ cohort ( 50 meiosis), i.e. the most extreme high and low responders to intratracheal silica-instillation. a)-f): chromosomes 1-19; t): chromosome x. cM: centimorgans; $\cdots \cdot 0$; — : suggestive linkage threshold; - - -: significant linkage threshold. Putative QTL on chromosomes 3, 4, 6, 10,11, 14, and 18 identified by the genome scan were further analysed with the entire F2 cohort.

authors hypothesise that genetic background contributes significantly to the variation in inflammation and pulmonary fibrosis induced by inhalation of silica in the mouse.

First, to address this question, the relative susceptibility to silica exposure in inbred strains of mice was studied. The inbreeding process results in genetic homogeneity at almost all loci, and different inbred strains of mice may be homogeneous for different alleles at the same loci. Generally, strains with greater evolutionary divergence will have a greater degree of polymorphism than strains that are closely related. Therefore, these characteristics enable investigations into genetic basis for a physiological and/or toxicological response of a phenotype. That is, if a difference in a chosen phenotype is found after a screen of a number of inbred strains to provide sufficient variation across the species, then it may be concluded that one or more loci contributes to the genetic variance observed among strains [17]. The present study evaluated fibrotic response after exposure to intratracheal silica-instillation in eight strains of mice chosen based on their differing lineage and common usage in genetic studies. The significant interstrain variation (genetic) in the fibrotic response to silica exposure supports the hypothesis that susceptibility to silica exposure in inbred mice has a significant genetic component.

Secondly, to determine whether susceptibility to silica exposure is inherited as a dominant or recessive trait, hydroxyproline content was determined in silica-exposed B6CBAF1/J mice. Although the hydroxyproline content of B6CBAF1/J mice was different to that of silica-exposed C57BL/6J and 

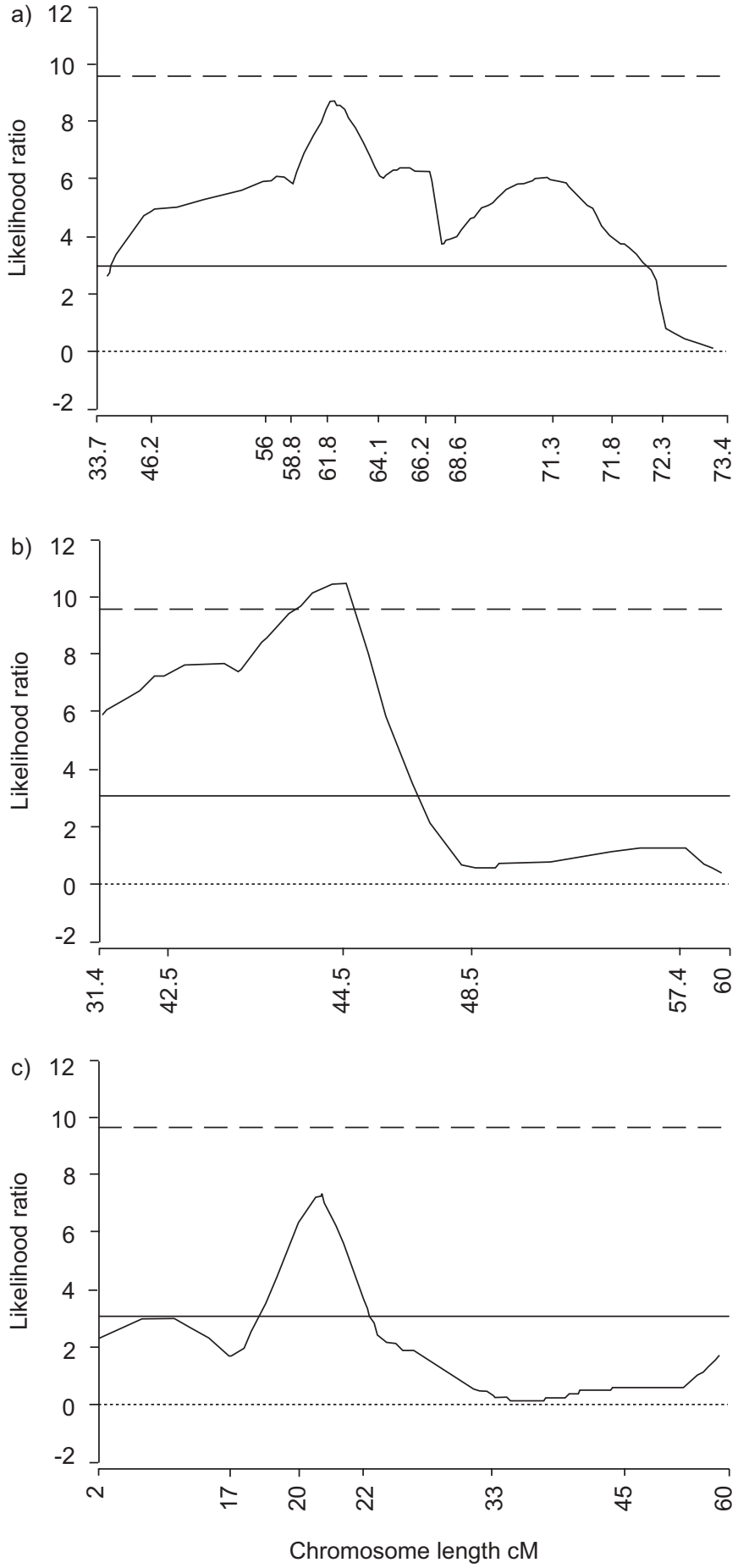

FIGURE 5. Chi-squared likelihood ratio for the association of silica-instilled, lung hydroxyproline content (\% of controls) phenotype with polymorphic simple sequence length polmorphism markers on chromosomes a) 3, b) 4 and c) 18 . Internal mappings were performed by simple linear regression, — : suggestive linkage threshold; - - -: significant linkage threshold.

CBA/J mice, the phenotype of F1 showed full phenotype of the dominant trait and the mode of inheritance seems to be complex in this model. Further characterisation of the mode of inheritance will need formal segregation analyses in segregated backcross and intercross populations derived from
C57BL/6J and CBA/J mice as previously performed by KLEEBERGER et al. [18].

Thirdly, to further explore a genetic basis for susceptibility to silicosis, a QTL analysis was performed of a large F2 mice population generated from the CBA/J and C57BL/6J strains. In this study, a significant QTL for susceptibility to silicosis was identified on chromosome 4 with two suggestive QTLs also identified on chromosomes 3 and 18 .

Several studies have used inbred strains of mice to identify susceptible genes for pulmonary fibrosis. HASTON and coworkers $[19,20]$ identified two genetic loci, chromosome 17 (MHC) and chromosome 11 in a radiation-induced lung fibrosis model. These QTL nearly overlapped QTL identified for ozone and particular matter susceptibility [19, 21], leading HASTON et al. [22] to propose a common genetic influence in mouse models of lung fibrosis.

In the current study, a significant QTL was identified on chromosome 4 , with a peak likelihood ratio occurring at the microsatellite marker D4Mit9.1 (located $44.5 \mathrm{cM}$ distal to the centromere) and two suggestive QTLs identified in chromosomes 3 and 18. These QTL were different from other QTL in a mouse model of bleomycin- and radiation-induced lung fibrosis. The present results suggest that different mechanisms may play roles in pathophysiological mechanisms in the development of lung fibrosis. Differing from bleomycin and radiation, crystalline of silica is an inorganic dust and causes a granulomatous reaction in the lung. This factor might induce different pathophysiological mechanisms in the lung and yield different results.

There are several candidate genes within chromosome 4 QTL, including the oncogene $j u n$, insulin-like growth factor binding protein-like-1 (Igfbp-1), phospholipase A2 activating protein plaa, and cyclin-dependent kinase $c d k n 2 a$. The oncogene jun has transcriptional regulatory roles with $\mathrm{p} 21$, and is involved in cell mitogenic responses and growth [23]. Insulin-like growth factor binding proteins (IGFBPs), as well as insulin growth factors (IGFs), have been proposed as pathogenic in lung fibrosis. Some IGFBPs work as modulating factors of IGF and have also been detected in idiopathic pulmonary fibrosis tissues [24]. Although the function of $I g f b p-1$ has not been clarified with respect to the current data, it may have potential to be one of several important candidate genes. Other candidate genes, such as plaa and $c d k n 2$, are also transcriptional factors and have important roles in cell cycle machinery. Candidate genes around the QTL on chromosome 18 include fibroblast growth factor-1 (fgf-1) sprouty homolog 4 (Spry4; Drosophilia), and pulmonary adenoma susceptibility-9 (pas9). Functions of $f g f-1$ have been implicated in the hepatic fibrotic process [25]. Spry4 is an intracellular fibroblast growth factor receptor antagonist, expressed in epithelial cells of foetal lung under control of a doxycycline-inducible system. As these factors are all suggested in silicosis, their function in silicosis models warrants further investigation.

In a previous study by OHTSUKA et al. [9], the inheritance of phenotypic traits suggests that this silicosis model is a polygenetic rather than a single genetic model. In the present study, the sum of total trait variance explained by the three 
QTL is $23 \%$. These results suggest the important contribution of genetic factors in response to instilled silica.

In conclusion, significant inter-strain variation was shown to exist in the pulmonary responses to intratracheal instillation of silica. From this, the present authors conclude that there is a significant genetic effect on fibrotic response to silica. Furthermore, the genome-wide linkage analysis of F2 identified significant quantitative trait loci for silicosis on chromosome 4 and suggestive quantitative trait loci on chromosomes 3 and 18, respectively. This is the first demonstration of candidate loci for susceptibility to silicosis. Several interesting candidate genes (e.g. $f g f$, igfbpl-1) have been observed within the regions of interest and polymorphisms within these genes need to be further analysed. Future approaches will be the comparative sequencing of the candidate genes in eight strains of mice. To refine the identified quantitative trait loci will provide an understanding of the mechanism of the host response to silica exposure and provide a potential means to identify genetically susceptible individuals who may be at risk to adverse effects of silica exposure.

\section{REFERENCES}

1 National Institute for Occupational Safety and Health. Work-related lung disease surveillance report. Publication No. 2000-105. Cincinnati (OH), DHHS (NIOSH), 1999.

2 American Thoracic Society. Adverse effect of crystalline silica exposure. Am J Respir Crit Care Med 1997; 155: 761-765.

3 Honda K, Kimura A, Dong RP, et al. Immunogenetic analysis of silicosis in Japan. Am J Respir Cell Mol Biol 1993; 8: 106-111.

4 Rihs HP, Lipps P, May-Taube K, et al. Immunogenetic studies on HLA-DR in German coal miners with and without coal worker's pneumoconiosis. Lung 1994; 172: 347-354.

5 Corbett E, Mozzato-Chamay N, Butterworth AE, et al. Polymorphisms in the tumor necrosis factor- $\alpha$ gene promoter may predispose to severe silicosis in black South African miners. Am J Respir Crit Care Med 2002; 165: 690-693.

6 Yucesoy B, Vallyathan V, Landsittel DP, et al. Association of tumor necrosis factor- $\alpha$ and interleukin-1 gene polymorphisms with silicosis. Toxicol Appl Pharmacol 2001; 172: 75-82.

7 Yucesoy B, Vallyathan V, Landsittel DP, et al. Polymorphisms of the IL-1 gene complex in coal miners with silicosis. Am J Industrial Med 2001; 39: 286-291.

8 Ohtsuka Y, Munakata M, Ukita $\mathrm{H}$, et al. Increased susceptibility to silicosis and TNF-alpha production in C57BL/6J mice. Am J Respir Crit Care Med 1995; 152: 2144-2149.

9 Ohtsuka Y, Wang X, Saito J, Ishida T, Munakata M. Genetic linkage analysis of susceptibility to silica exposure in mice. Am J Respir Crit Care Med 2004; 169: A526.
10 Ashcroft T, Simpson JM, Timbrell V. Simple method of estimating severity of pulmonary fibrosis on a numerical scale. J Clin Pathol 1988; 41: 467-470.

11 Woessner JF Jr. The determination of hydroxyproline in tissue and protein samples containing small proportions of this amino acid. Arch Biochem Biophys 1961; 93: 440-447.

12 Informative markers for a cross - mouse microsatellite studies. http://www.cidr.jhmi.edu/mouse/mouse_dif.html. Date last updated: October 2005. Date last accessed: November 2005.

13 Silver LM. Mouse Genetics. Concepts and Applications. New York, Oxford University Press, 1995.

14 Manly KF, Cudmore Jr RH, Meer JM. Map Manager QTX, cross-platform software for genetic mapping. Mammalian Genome 2001; 12: 930-932. http:/ /www.mapmanager.org/ mmQTX.html. Date last accessed: November 2005.

15 Churchill GA, Doerge RW. Empirical threshold values for quantitative trait mapping. Genetics 1994; 138: 963-971.

16 Lander ES, Kruglyak L. Genetic dissection of complex traits: guidelines for interpreting and reporting linkage results. Nat Genet 1995; 11: 241-227.

17 Kleeberger SR, Ohtsuka Y. Gene-particulate matter-health interactions. Toxicol Appl Pharmacol 2005; 207: Suppl. 2, 276-281.

18 Kleeberger SR, Levitt RC, Zhang LY, et al. Linkage analysis of susceptibility to ozone-induced lung inflammation in inbred mice. Nat Genet 1997; 17: 475-478.

19 Haston CK, Travis EL. Murine susceptibility to radiationinduced pulmonary fibrosis is influenced by a genetic factor implicated in susceptibility to bleomycininduced pulmonary fibrosis. Cancer Research 1997; 57: 5286-5291.

20 Haston CK, Wang M, Dejournett RE, et al. Bleomycin hydrolase and a genetic locus within the MHC affect risk for pulmonary fibrosis in mice. Hum Mol Genet 2002; 11: 1855-1863.

21 Ohtsuka Y, Brunson KJ, Jedlicka AE, et al. Genetic linkage analysis of susceptibility to particle exposure in mice. Am J Respir Cell Mol Biol 2000; 22: 574-581.

22 Haston C K, Zhou X, Gumbiner-Russo L, et al. Universal and radiation-specific loci influence murine susceptibility to radiation-induced pulmonary fibrosis. Cancer Research 2002; 62: 3782-3788.

23 Shaulian E, Schreiber M, Piu F, Beeche M, Wagner EF, Karin M. The mammalian UV response: c-Jun induction is required for exit from p53-imposed growth arrest. Cell 2000; 103: 897-900.

24 Pilewski JM, Liu L, Henry AC, Knauer AV, FeghaliBostwick CA. Insulin-like growth factor binding proteins 3 and 5 are overexpressed in idiopathic pulmonary fibrosis and contribute to extracellular matrix deposition. Am J Pathol 2005; 166: 399-407.

25 Yu C, Wang F, Jin C, et al. Role of fibroblast growth factor type 1 and 2 in carbon tetrachloride-induced hepatic injury and fibrogenesis. Am J Pathol 2003; 163: 1653-1662. 